ANNALES

POLONICI MATHEMATICI

XL (1981)

\title{
On S. Goląb's characterization of the anharmonic ratio
}

\author{
by Bogdan Choczewski (Kraków)
}

\begin{abstract}
S. Gołąb [5] proposed a system of functional equations in several variables to characterize the anharmonic ratio of four points of the projective line. In this paper we replace one of $\mathbf{S}$. Goląb's conditions by a weaker one, which leads to a functional equation in a single variable, and we prove that the anharmonic ratio is also the only function fulfilling our conditions.
\end{abstract}

1. The anharmonic (cross) ratio of a quadruple of colinear points of the projective space can be characterized with the aid of functional equations. This has been first done by J. Aczél, S. Goląb, M. Kuczma and E. Siwek [1], who solved the functional equation

$$
\Phi\left(T\left(x_{1}\right), T\left(x_{2}\right), T\left(x_{3}\right), T\left(x_{4}\right)\right)=\Phi\left(x_{1}, x_{2}, x_{3}, x_{4}\right),
$$

where $x_{i}$ are the affine coordinates of the four points on the projective line $L$, and $T: L \rightarrow L$ denotes any homography: $x \mapsto T(x)=(a x+b) /(c x+d)$, $a d-b c \neq 0$. The authors proved, without any regularity assumptions on $\Phi$, that solutions to (*), i.e. four-point-invariants under the projective transformations of the line, are all functions of the anharmonic ratio.

This result was generalized in different directions. W. Benz [3] considered (*) in the case of the projective group of transformations acting on the projective line over a skew field. A more general equation corresponding to (*) but written in homogeneous coordinates has been solved by $\mathrm{S}$. Topa [10].

Recently H. Schwerdtfeger [8] pointed out that the essential point in equation (*) is that the group $G_{3}$ of homographies $T$ is exactly three-fold transitive (i.e., for every $x_{i}$ and $y_{i}$ arbitrarily chosen on $L$ there is the unique mapping $T \in G_{3}$ to fulfil $\left.T\left(x_{i}\right)=y_{i} ; i=1,2,3\right)$. He obtained the same result as that mentioned for (*) but regarding an exactly $n$-fold transitive group $G_{n}$. Such invariants have also been dealt with by W. Benz [4].

2. Another characterization of the anharmonic ratio by means of 
functional equations has been proposed by S. Gołąb [5], who considered for a mapping $S: D \rightarrow R$, where

$$
D:=\left\{\left(x_{1}, x_{2}, x_{3}, x_{4}\right): x_{i} \neq x_{j} \text { for } i \neq j\right\},
$$

the following system of equations:

$$
\begin{gathered}
S\left(x_{3}, x_{4} ; x_{1}, x_{2}\right)=S\left(x_{1}, x_{2} ; x_{3}, x_{4}\right), \\
S\left(x_{1}, x_{3} ; x_{2}, x_{4}\right)=1-S\left(x_{1}, x_{2} ; x_{3}, x_{4}\right), \\
S\left(x_{1}, x_{2} ; x_{3}, x_{4}\right) S\left(x_{1}, x_{2} ; x_{4}, x_{5}\right)=S\left(x_{1}, x_{2} ; x_{3}, x_{5}\right) .
\end{gathered}
$$

S. Gołąb proved in [5] that the general solution of system (2)-(4) is given by the formula

$$
S\left(x_{1}, x_{2} ; x_{3}, x_{4}\right)=s\left(\alpha\left(x_{1}\right), \alpha\left(x_{2}\right) ; \alpha\left(x_{3}\right), \alpha\left(x_{4}\right)\right),
$$

where $\alpha: R \rightarrow R$ is an arbitrary injection on $R$ and $s$ is given by

$$
s\left(x_{1}, x_{2} ; x_{3}, x_{4}\right)=\left(x_{1}-x_{3}\right)\left(x_{2}-x_{4}\right) /\left[\left(x_{2}-x_{3}\right)\left(x_{1}-x_{4}\right)\right]
$$

for $\left(x_{1}, x_{2}, x_{3}, x_{4}\right) \in D$, i.e. denotes the anharmonic ratio. The solution has been found without any regularity assumptions on $S$.

The question arises under what additional assumptions on $S$ it becomes the anharmonic ratio $s$. S. Goląb answered the question in [5], proving that if the function $\alpha$ in (5) is strictly monotonic in $R$ and if we have

$$
S(x, y ; 2 x y /(x+y), 0)=-1 \quad \text { for }|x| \neq|y|, x \neq 0,
$$

then necessarily $S=s$.

Condition (7) means that $S=s$ on the two-parameter family of harmonic points of the projective line $L$.

In the present paper we shall assume that $S=s$ on a one-parameter family of harmonic points on $L$, viz. that

$$
S(x, 1 ; 2 x /(x+1), 0)=-1 \quad \text { for } x \in R^{\prime}:=R \backslash\{-1,0,1\},
$$

and, using the theory of iterative functional equations, we shall prove, in particular, that if the $\alpha$ in (5) is injective and of class $C^{1}$ in a neighbourhood of $x=1$, then $S=s$ on $D$. Some weaker conditions to the effect will be also given.

3. We shall look for solutions $\alpha$ of (8) which are homographies. Since $s$ is invariant under these transformations, this will imply $S=s$ in $D$, by (5).

Let us first write equation (8) for $\alpha$ (using (5) and (6)) and transform it to a simpler form. This is ours

THEOREM 1. If the function $S$ of form (5)-(6) with an injective $\alpha: R \rightarrow R$ fulfils equation (8) in $R^{\prime}$, then the function

$$
\varphi(x):=b^{-1}+[\alpha(x)-\alpha(0)]^{-1} \text { for } x \neq 0 ; \varphi(0):=b^{-1},
$$


where

$$
b:=\alpha(0)-\alpha(1),
$$

is an injective map of $R$ into itself, fulfilling the equation

$$
\varphi(2 x /(x+1))=\frac{1}{2} \varphi(x), \quad x \in R^{\prime} .
$$

Conversely: if $\varphi: R \rightarrow R$ is injective and it fulfils (11) in $R^{\prime}$, then the function $S$ given by (5)-(6) with the $\alpha: R \rightarrow R$ defined by the formula

$$
\alpha(x):=a+[\varphi(x)-\varphi(0)]^{-1} \text { for } x \neq 0 ; \alpha(0):=a,
$$

with an arbitrary $a \in R$, satisfies equation (8).

Proof. Inserting (5)-(6) to (8) we get the equation

$$
[\alpha(g(x))-\alpha(x)][\alpha(0)-\alpha(1)]=[\alpha(g(x))-\alpha(1)][\alpha(x)-\alpha(0)]
$$

for $x \in R^{\prime}$, where we put

$$
g(x):=2 x /(x+1), \quad x \neq-1 .
$$

Denoting $a:=\alpha(0)$, so that $\alpha(1)=a-b$ (cf. (10)), and

$$
\beta(x):=\alpha(x)-a, \quad x \in R,
$$

we can rewrite (13) in the form

$$
[\beta(g(x))-\beta(x)] b={ }^{\prime}[\beta(g(x))+b] \beta(x)
$$

for $x \in R^{\prime}$, i.e.

$$
\beta(g(x)) \beta(x)-b \beta(g(x))+2 b \beta(x)=0 .
$$

This means that the function

$$
\varphi(x):=1 / \beta(x)+1 / b \quad \text { for } x \neq 0 ; \varphi(0):=1 / b,
$$

fulfils equation (11) in $R^{\prime}$. Formulae (16) and (15) yield (9) for the function $\varphi$.

Conversely, given an injective solution $\varphi: R \rightarrow R$ of equation (11) in $R^{\prime}$, we necessarily have $\varphi(1)=0$. Calculating $\beta$ from (15) with an arbitrary $a \in R$, we check that the $\alpha$ has the form (12) and that it fulfils (13). Equation (13) implies (8) for the function $S$ given by (5)-(6).

This completes the proof of the theorem.

Thus all the injective solutions $\alpha$ of (13) in $R^{\prime}$ are compositions of a homography with a solution $\varphi$ of (11) (in $R^{\prime}$ ).

4. By the results of the preceding section we are led to the study of injective solutions of the Schröder equation (11).

We shall make use of two theorems on the existence of a unique one parameter family of solutions to the Schröder functional equation

$$
\psi(f(x))=p \psi(x), \quad 0<p<1 .
$$


First of all we assume that

(H) The function $f:[0, b) \rightarrow[0, b)$ is strictly increasing and continuous in $[0, b)$ and $0<f(x)<x$ in $(0, b)$.

Denote by $f^{n}$ the $n$-th iterate of the function $f$. The following two theorems can be found in M. Kuczma's book [7].

LEMMA 1 ([7], Theorem 6.8, p. 143). If the function $f$ fulfils hypotheses $(\mathrm{H})$, and it is convex in $[0, b), f^{\prime}(0)=p\left({ }^{1}\right)$, then there is a unique one-parameter family of convex solutions of $(17)$ in $[0, b)$, given by the formula.

$$
\psi(x)=c \lim _{n \rightarrow \infty}\left[f^{n}(x) / f^{n}\left(x_{0}\right)\right]
$$

where $c \in R$ and $x_{0}$ is an arbitrary point in $(0, b)$. These solutions are continuous and for $c \neq 0$ strictly monotonic in $[0, b)$.

Remark 1. The lemma remains true if we replace the interval $[0, b)$ by the open interval $(0, b)$. The existence of $f^{\prime}(0)$ must then be additionally assumed.

Lemma 2 ([9], of. also [7], Theorem 6.3 and 6.2, p. 137-138). Let $f$ fulfil $(\mathrm{H})$ and be of class $C^{1}$ in $[0, b)$. If there is a positive number $r$ fulfilling

$$
f^{\prime}(x)=p+O\left(x^{r}\right), \quad x \rightarrow 0+,
$$

then there exists a unique one-parameter family of $C^{1}$-solutions of (17) in $[0, b)$, given by the formula

$$
\psi(x)=c \lim _{n \rightarrow \infty} p^{-n} f^{n}(x) .
$$

These solutions are strictly monotonic in $[0, b)($ for $c \neq 0)$.

We shall also need a theorem on extension of solutions of (17). The lemma which follows is a consequence of a general extension theorem due to $K$. Baron [2]. It can be also derived from a theorem on the form of the general solution of the Schröder equation, which has been proved by M. Kuczma ([7], Theorem 1.10 , p. 43).

Lemma 3. Let $f: A \rightarrow A$, where $A \supset[0, b)$ is a set, be a function whose restriction to the interval $[0, b)$ satisfies Hypotheses $(\mathbf{H})$. If $\psi_{0}:[0, b) \rightarrow R$ is a solution of $(17)$ in $[0, b)$, then there exists exactly one solution $\psi: A \rightarrow R$ of (17) in $A$, such that $\psi(x)=\psi_{0}(x)$ for $x \in[0, b)$.

5. We return to equation (11), i.e. to the equation

$$
\varphi(g(x))=\frac{1}{2} \varphi(x)
$$

where the function $g$ is defined by (14). This function has two fixed points: repulsive $x=0$ and attractive $x=1$ (that means, there is a neighbourhood

( ${ }^{1}$ The derivative actually exists since $f$ is convex in $[0, b)$. 
of $x=1$ such that $f^{n}(x) \rightarrow 1$ in it and $f^{n}(x)$ never tends to zero if $x \neq 0$; cf. [7], p. 18).

We shall look for solutions of (21) in a neighbourhood of $x=1$. In order to be able to apply the results collected in Section 4 we need to move this point to the origin. Using the transformation

$$
g(x) \mapsto f(x):=g(x+1)-1,
$$

we get a function, which has the attractive fixed point $x=0$. We have

$$
f(x)=x /(x+2) \quad \text { for } x \neq-2
$$

and, instead of (21), the equation

$$
\psi(f(x))=\frac{1}{2} \psi(x)
$$

A function $\psi$ is a solution of (23) in a set $A$ iff the function

$$
\varphi(x)=\psi(x-1)
$$

satisfies (21) on the shifted set $A+1$.

We shall deal with equation (23) in an interval $[0, b), b>0$.

THEOREM 2. Equation (23) with the function (22) restricted to the interval $[0, b)$ has only the one-parameter family of solutions $\psi$ both in the class of convex (or concave) functions in $[0, b)$ and in the class $C^{1}[0, b)$. The solutions are given by the formula

$$
\psi(x)=d x /(x+1), \quad x \in[0, b), d \in R .
$$

Proof. It is enough to verify the assumptions of Lemmas 1 and 2 . Our function $f$ given by (22) obviously fulfils $(\mathrm{H})$ in every interval $[0, b)$ and it is convex there, $f^{\prime}(0)=\frac{1}{2}=p$ (the $p$ in (23)). Thus Lemma 1 implies that all the convex solutions of (23) are given by (18), where

$$
f^{n}(x)=2^{-n} x\left(1+\left(1-2^{-n}\right) x\right)^{-1} \text {. }
$$

Calculating the limit (18), we get (25) with $d=c\left(x_{0}+1\right) / x_{0}$.

Similarly we see that $f$ is of class $C^{1}[0, b)$ and

$$
f^{\prime}(x)=2(x+2)^{-2}=\frac{1}{2}+O(x), \quad x \rightarrow 0+,
$$

i.e. (19) is fulfilled with $r=1$. By Lemma 2 all the $C^{1}$-solutions of (23) in $[0, b)$ are then given by formula $(20)$, with $p=\frac{1}{2}$ and $f^{n}(x)$ from (26). This again yields (25), with $d=c$.

Let us complete the definition of the function $f$ requiring it to be an injective map of $(-\infty,+\infty]$ onto itself. We put

$$
f(+\infty)=1, \quad f(-2)=+\infty .
$$

Theor em 3. All the solutions $\psi: A \rightarrow R$, where $A:=(-\infty,+\infty] \backslash\{-1\}$, 
of equation (23) (with the function $f$ defined by (22) and (27)), whose restrictions to an interval $[0, b)$ are either convex functions or $C^{1}$-functions, are given by the formula

$$
\psi(x)=d x /(x+1) \quad \text { for } x \in R \backslash\{-1\}, \psi(+\infty)=d .
$$

Proof. The functions (28) actually fulfil equation (23) in $R \backslash\{-2,-1\}$. Let $x=-2$. Then $f(-2)=+\infty$, by (27), and $\psi(-2)=2 d, \psi(f(-2))$ $=\psi(+\infty)=d$, by (28). Thus (23) holds for $x=-2$. We have also $\psi(f(+\infty))=\psi(1)=\frac{1}{2} d=\frac{1}{2} \psi(+\infty)$, i.e. (23) for $x=+\infty$.

On the other hand, since the function $f$ defined by (22) and (27) maps the set $A$ onto itself, we can apply Lemma 3 . This lemma says that there exists a unique extension to $A$ of every solution of $(23)$ in $[0, b)$. Functions (28) are such extensions of functions (25). They fulfil (23) in $A$, as we have checked, and there is no other solution than (25) in the classes of functions considered (Theorem 2). This completes the proof.

6. Now we can return to the problem of the characterization of the anharmonic ratio. We shall prove the following

THEOREM 4. Let the function $S: D \rightarrow R$ (cl. (1)), of form (5)-(6) with an injective $\alpha: R \rightarrow R$, fulfil equation (8) in $R^{\prime}$. Then there exists such an injective $\varphi: R \rightarrow R$, that the $\alpha$ is given by (12) and $\varphi$ satisfies equation (11) in $R^{\prime}$.

On the other hand, for every injective $\varphi: R \rightarrow R$ satisfying (11) in $R^{\prime}$ and such that the restriction $\varphi_{1}:=\left.\varphi\right|_{(1,1+b)}$ is a function either convex or of class $C^{1}$ in $[1,1+b)$, the corresponding function $\alpha$, defined by (12), is a homography. Consequently, $S=s-$ where $s$ is the anharmonic ratio (6).

Proof. The first part of the theorem results from Theorem 1. Further, solutions $\bar{\varphi}$ of equation (11) in $R^{\prime}$, whose restrictions $\bar{\varphi}_{1}$ possess the required properties, are of the form

$$
\bar{\varphi}(x)=d(x-1) / x, \quad x \in R \backslash\{0\} .
$$

This follows from (24) and (28) of Theorem 3. (Note that functions (28) satisfy equation (23) in the set $A$ containing $R^{\prime}$.) Let us put

$$
\varphi(x)=d(x-1) / x \quad \text { for } x \neq 0 ; \varphi(0):=d, d \neq 0 .
$$

Functions (29) are the only injections defined in $R$ and such that the functions $\left.\varphi\right|_{R^{\prime}}$ satisfy equation (11) in $R^{\prime}$.

Using (29) to determine the functions $\alpha$ by (12), we get

$$
\alpha(x)=a-\frac{d}{x} \quad \text { for } x \neq 0 ; \alpha(0)=a,
$$

i.e. $\alpha$ is an injective map of $R$ into itself. Moreover, the $\alpha$ is a homography. This yields $S=s$ on $D$, according to the introductory remarks in Section 3 above. This ends the proof of the theorem. 
Remark 2. The simplest consequence of Theorem 4 can be formulated as follows:

COROllary. If the function $S: D \rightarrow R$ is of form (5)-(6), fulfils (8) in $R^{\prime}$ and if the function. $\alpha$ is injective and of class $C^{1}$ in a neighbourhood of $x=1$, then $S=s$.

Proof. This follows from Theorem 4 and formulae (12) and (9): if $\alpha$ is a $C^{1}$-function in a neighbourhood of $x=1$, then so is also the function $\varphi$, and conversely.

Remark 3. A stronger result than those contained in Theorem 4 is obtained by assuming that the function $\varphi_{1}$ has the property: " $\left[\varphi_{1}(x)-1\right] /(x-1)$ is monotonic in $(1,1+b)$ ". This results from a uniqueness theorem due to M. Kuczma [6], which is more general than that quoted as Lemma 1. The theorem reads: "If $f$ satisfying Hypotheses $(\mathrm{H})$ is such that the function $f(x) / x$ is monotonic in $(0, b)$ and $\lim _{x \rightarrow 0^{+}} f(x) / x=p$, then formula (18) yields all the solutions of (17) such that $\psi(x) / x$ is monotonic in $(0, b)$; and only these." Observe that the function $f$ given by (22) actually has the above required properties. Thus formula (28) remains valid in the class of functions for which the ratio $\psi(x) / x$ is monotonic in a neighbourhood of the origin. Consequently, (30) also holds true.

\section{References}

[1] J. Aczél, S. Gołąb, M. Kuczma and E. Siwek, Das Doppelverhältnis als Lösung einer Funktionalgleichung, Ann. Polon. Math. 9 (1960), p. 183-187.

[2] K. Baron, On extending solutions of a functional equation, Aequationes Math. 13 (1975), p. 285-288.

[3] W. Benz, Die 4-Punkt-Invarianten in der projektiven Geraden liber einem Schiefkörper, Ann. Polon. Math. 21 (1968), p. 97-101.

[4] - The n-point-invariants of the projective line and cross ratio of n-tuples, ibidem 26 (1972), p. 53-60.

[5] S. Gołąb, Sur une système d'équations fonctionnelles lié au rapport anharmonique, ibidem 29 (1974), p. 273-280.

[6] M. Kuczma, Note on Schröder's functional equation, J. Austral. Math. Soc. 4 (1964), p. 149-151.

[7] - Functional equations in a single variable, PWN, Warszawa 1968.

[8] H. Schwerdtfeger, Invariants of a class of transformation groups, Aequationes Math. 14 (1976), p. 105-110.

[9] G. Szekeres, Regular iteration of real and complex functions, Acta Math. 100 (1958), p. 203-258.

[10] S. Topa, On a generalization of the functional equation for the harmonic ratio of four points on a projective line over an arbitrary commutative field, Ann. Polon. Math 23 (1970), p. $65-72$. 